\title{
Droplet formation in microfluidic T-junction generators operating in the transitional regime. III. Dynamic surfactant effects
}

\author{
Tomasz Glawdel and Carolyn L. Ren \\ Department of Mechanical and Mechatronics Engineering, University of Waterloo, Waterloo, Ontario N2L 3G1, Canada
}

(Received 17 April 2012; published 13 August 2012)

\begin{abstract}
This study extends our previous work on droplet generation in microfluidic T-junction generators to include dynamic interfacial tension effects created by the presence of surfactants. In Paper I [T. Glawdel, C. Elbuken, and C. L. Ren, Phys. Rev. E 85, 016322 (2012)], we presented experimental findings regarding the formation process in the squeezing-to-transition regime, and in Paper II [T. Glawdel, C. Elbuken, and C. L. Ren, Phys. Rev. E 85, 016323 (2012)] we developed a theoretical model that describes the performance of T-junction generators without surfactants. Here we study dynamic interfacial tension effects for two surfactants, one with a small molecular weight that adsorbs quickly, and the other with a large molecular weight that adsorbs slowly. Using the force balance developed in Paper II we extract the dynamic interfacial tension from high speed videos obtained during experiments. We then develop a theoretical model to predict the dynamic interfacial tension in microfluidic T-junction generators as a function of the surfactant properties, flow conditions, and generator design. This model is then incorporated into the overall model for generator performance to effectively predict the size of droplets produced when surfactants are present.
\end{abstract}

DOI: 10.1103/PhysRevE.86.026308

PACS number(s): 47.61.Jd, 47.55.db

\section{INTRODUCTION}

This study focuses on the influence of surfactants on the production of droplets in microfluidic generators. Surfactants are often added to reduce the interfacial tension and prevent unwanted coalescence of droplets [1-3]. However, their presence adds additional complexity to the emulsification process as there exists intensive coupling between the mass transport of surfactant and the droplet formation process through the interfacial tension. Surfactant adsorption to the interface depends on the characteristics of the surfactant (concentration, diffusion coefficient, micelle kinetics) and the mass transfer process including convection, diffusion, and dilation rate of the interface. Therefore, during the formation process the interfacial tension changes dynamically within the range bounded by the equilibrium of the pure liquids and the system with the added surfactant. Thus it is important to study these dynamic effects on the production of droplets in microfluidic generators.

In fact, several techniques have been developed that utilize droplet formation at a small orifice (usually a capillary tube) to measure the adsorption process through the dynamic interfacial tension; examples include the drop volume method [4-6], maximum bubble pressure method [7-9], and growing drop method [10,11]. Generally, these techniques analyze the formation at rather large orifices (250-1000 $\mu \mathrm{m})$ and longer time scales $(>0.5 \mathrm{~s})$ in comparison to the typical size $(20-100 \mu \mathrm{m})$ and time scale $(0.001-0.5 \mathrm{~s})$ of microfluidic emulsification. Thus data obtained with these methods are not immediately transferrable to microfluidic applications.

A more apt comparison to microfluidic emulsifications is membrane emulsifications where droplets are also generated from small pores $(5-50 \mu \mathrm{m})$ at high rates $(\sim 1 \mathrm{kHz})[12,13]$. A considerable amount of work has documented the effects of surfactant dynamics on the production of droplets from membranes including the relationship between dynamic interfacial tension and the size of droplets [14-17], the lag time between consecutive droplets [18], and the number of active pores. In addition to the influence of the surfactant properties, these studies revealed that the dispersed phase flux also has an important influence as it determines the degree of surfactant depletion at the interface from surface expansion.

A few works have studied dynamic interfacial tension effects in microfluidic droplet generators. Experiments by van der Graaf et al. [14] with T-junction generators demonstrated that under low surfactant concentration, droplet formation is comparable to when surfactants are absent, indicating that droplet production is faster than surfactant adsorption in most cases. Only at high concentration of surfactants was a change in droplet production seen with smaller droplets being produced. Baret et al. studied the influence of surfactant kinetics on droplet stabilization by directly monitoring the adsorption of surfactants to the interface using fluorescently labeled surfactants [19]. Experiments showed that the interface is nearly bare after the droplet is produced and coverage increases with time as the droplet travels down the microchannel. Coverage across the droplet is also nonuniform as surfactant accumulates near the back end of the droplet where a stagnation point exists.

Adsorption kinetics have also been studied through the measurement of droplet production in microfluidic generators [20-23]. Wang et al. used droplet size, and Nguyen et al. used frequency to quantify the dynamic interfacial tension with microfluidic T-junction generators [22,23]. Both of these studies focused on formation times in the millisecond range. Steegmans et al. looked at submillisecond effects by analyzing droplet production in a shallow Y-channel design with high flow rates [20]. These studies used semiempirical models to infer the interfacial tension from calibration curves first obtained with pure liquid-liquid combinations. Generally, the measured interfacial tension was far from the equilibrium value, even for small surfactants that were expected to adsorb quickly to the interface.

In Paper I [24] of this study we presented experimental observations on the operation of microfluidic T-junction droplet generators operating in the squeezing-to-transition 
regime. Through analysis of high speed videos we divided the formation process into three stages: (i) a lag stage where the interface recedes back into the side channel a distance $L_{\text {lag }}$ after the detachment of the preceding droplet, (ii) a filling stage where the droplet penetrates into the cross-flowing stream to a depth $b_{\text {fill }}$ as it is deformed by pressure forces, and (iii) a necking stage where the pressure forces overwhelm the droplet leading to the eventual detachment of the droplet once the neck reaches a critical thickness $2 r_{\text {pinch }}$. We investigated the dependence of these parameters on the geometry of the T-junction generator, including the ratio of the dispersed to continuous channel widths $\Lambda^{*}=w_{d} / w_{c}$, the height to width ratio of the channels $h^{*}=h / w_{c}$, the flow ratio and viscosity ratio of the two phases $\varphi=Q_{d} / Q_{c}$ and $\eta=\mu_{d} / \mu_{c}$, and the capillary number $\mathrm{Ca}=\mu_{c} U / \gamma$-with $U$ and $\mu_{c}$ the average velocity and viscosity of the continuous phase, and $\gamma$ the interfacial tension.

In Paper II [25], a theoretical model was developed to describe the operation of the T-junction generator in order to predict the size, spacing, and frequency of formation. The equation that describes the volume of droplets produced has the form $V_{\text {drop }} / w_{c}^{2} h=\alpha_{\text {lag }}+\alpha_{\text {fill }}+\beta \varphi$, where the volume is normalized by $w_{c}^{2} h, \alpha_{\text {lag }}$ and $\alpha_{\text {fill }}$ are the normalized volume added during the lag and filling stages, and $\beta$ is the dimensionless necking time. As part of the model, mathematical expressions for these three parameters were developed based on theoretical arguments. The model was verified with data obtained across a range of T-junction geometries and flow conditions without the presence of surfactants.

Having demonstrated the successful modeling of droplet production in microfluidic T-junction generators operating in the transition regime, we now seek to examine the behavior when surfactants are present in the system. Using the same fluid combination as before, droplet formation is studied with the addition of surfactants by high speed video analysis. Two surfactants are studied, one with a small molecular weight that adsorbs quickly, and the other with a large molecular weight that adsorbs slowly. First, the surfactant experimental data are compared to the model developed in Paper II [25] to quantify the importance of dynamic interfacial tension. We then use the force balance within the model to extract the dynamic interfacial tension at the end of the filling stage and determine the influence of flow conditions and generator design on the process. Subsequently, a theoretical model is developed to account for the mass transfer of surfactant to the interface during the formation process and to predict the dynamic interfacial tension. We then incorporate the dynamic interfacial tension model into the previous model presented in Paper II [25] to predict overall generator performance in the presence of surfactants.

\section{METHODS AND MATERIALS}

The same experimental setup was used as described previously in Ref. [24]. Homogeneous poly(dimethyl)siloxane (PDMS) microchannnels were fabricated using standard soft lithography methods. Fluid flow was controlled using a microfluidic pressure system and videos were taken with a high speed camera. Experiments were limited to only two T-junction designs to test the influence of T-junction design
TABLE I. List of parameters covered in each experiment with surfactants.

Expt. No. Chip design $\left(\Lambda^{*}, h^{*}\right)$ Surfactant Concentration $(\% \mathrm{w} / \mathrm{v})$

\begin{tabular}{lccc}
\hline $1-3$ & $0.5,0.5$ & SDS & $0.5,1.5,3$ \\
$4-6$ & $0.5,0.5$ & SDS & $0.5,1.5,3$ \\
$5-9$ & $1,0.5$ & Tween 20 & $0.1,0.5,2$ \\
$10-12$ & $1,0.5$ & Tween 20 & $0.1,0.5,2$ \\
\hline \hline
\end{tabular}

on the dynamic interfacial tension. Channel height-to-width ratio was kept constant $\left(h^{*}=h / w_{c}=0.5\right)$ and the dispersed to continuous phase width ratio was varied $\left(\Lambda^{*}=w_{d} / w_{c}=\right.$ $0.5,1)$.

Experiments were performed with silicone oil as the continuous phase and different aqueous solutions containing surfactants as the dispersed phase. Two surfactants were chosen for the experiments: sodium dodecyl sulphate (SDS) and Tween 20, both of which are soluble in the aqueous phase. In particular, these two surfactants were chosen because they allow for a wide range of adsorption time scales to be investigated [22]. Three concentrations of each surfactant were tested: $0.5 \%, 1.5 \%$, and $3 \%(\mathrm{w} / \mathrm{v}) \mathrm{SDS}$, and $0.1 \%, 0.5 \%$, and $2 \%(\mathrm{w} / \mathrm{v})$ Tween 20 . All of these solutions exceed the critical micelle concentration by a significant degree. Premicellar solutions were not studied because (1) in most microfluidic applications high concentrations of surfactant are used to prevent coalescence of droplets in close quarters, and (2) at low concentrations, droplet production rates greatly exceed surfactant adsorption rates such that the interface remains essentially bare during droplet formation. Table I summarizes the parameters studied in each experiment. Different pressure combinations were applied to span a range of $\mathrm{Ca}$ and flow ratios for each experiment. Data were extracted from high speed videos in the same manner as described in Ref. [24].

The equilibrium interfacial tension for all combinations of the oil, water, and surfactant was measured using the Wilhemy Plate method (Data Physics DCAT 11). Without surfactant the equilibrium interfacial tension of pure watersilicone oil was measured as $38.75 \pm 0.01 \mathrm{mN} \mathrm{m}^{-1}$. For all Tween 20 combinations the equilibrium interfacial tension was $9.38 \pm 0.1 \mathrm{mN} \mathrm{m}^{-1}$ and for all SDS solutions it was $10.34 \pm 0.1 \mathrm{mN} \mathrm{m}^{-1}$. In addition, the viscosity of each solution was measured using a programmable rheometer (LVDV-III Ultra CPE, Brookfield Instruments). All solutions exhibited Newtonian behavior with minimal deviation from pure water ( 1 $\mathrm{mPa} \mathrm{s})$.

\section{A. Surfactant properties}

In modeling surfactant transport it is important to know the diffusion coefficient $(D)$ of the molecules, the critical micelle concentration $\left(c_{\mathrm{CMC}}\right)$, and the number of monomers contained in a micelle $\left(N_{A}\right)$. The reported diffusion coefficient for premicellar concentrations of SDS is approximately $8.0 \times$ $10^{-10} \mathrm{~m}^{2} \mathrm{~s}^{-1}$ [26]. Recent studies using the Fourier transform pulsed field gradient nuclear magnetic resonance (NMR) method decomposed the average diffusion coefficient into diffusion coefficients of $11.2 \times 10^{-10} \mathrm{~m}^{2} \mathrm{~s}^{-1}$ for monomers and $4.65 \times 10^{-11} \mathrm{~m}^{2} \mathrm{~s}^{-1}$ for dimers [27]. A CMC of 
TABLE II. Molecular formula, molecular weight $\left(M_{w}\right)$, critical micelle concentration $\left(c_{\mathrm{CMC}}\right)$, aggregation number $\left(N_{A}\right)$, and diffusion coefficient $(D)$ for the surfactants.

\begin{tabular}{lcc}
\hline \hline Surfactant & $\mathrm{SDS}$ & Tween 20 \\
\hline Molecular formula & $\mathrm{C}_{12} \mathrm{H}_{25} \mathrm{OSO}_{3} \mathrm{Na}$ & $\mathrm{C}_{58} \mathrm{H}_{114} \mathrm{O}_{26}$ \\
$M_{w}\left(\mathrm{~g} \mathrm{~mol}^{-1}\right)$ & 288.4 & 1227.2 \\
$c_{\mathrm{CMC}}\left(\mathrm{g} \mathrm{L}^{-1}\right)$ & $2.41^{\mathrm{a}}$ & $0.04^{\mathrm{b}}-0.138^{\mathrm{c}}$ \\
$N_{A}$ & $49.5-100^{\mathrm{d}}$ & $24-29^{\mathrm{e}}$ \\
$D\left(10^{-10} \mathrm{~m}^{2} \mathrm{~s}^{-1}\right)$ & $8.0^{\mathrm{f}}-11.2^{\mathrm{g}}$ & $0.3-1.9$ \\
\hline \hline
\end{tabular}

${ }^{\mathrm{a}}$ Reference [28].

${ }^{\mathrm{b}}$ Reference [34].

${ }^{\mathrm{c}}$ Reference [30].

${ }^{\mathrm{d}}$ Reference [28].

${ }^{\mathrm{e}}$ Reference [33].

${ }^{\mathrm{f}}$ Reference [26].

${ }^{\mathrm{g}}$ Reference [27].

$2.41 \mathrm{~g} \mathrm{~L}^{-1}$ was reported with an aggregate size between 49.5 and 100 monomers. Generally, micelle size and polydispersity increase with concentration above the CMC [28]. Tween 20, also known as Polysorbate 20, is a nonionic surfactant consisting of a complex mixture of oligomers with an average molecular weight of $1227.2 \mathrm{~g} \mathrm{~mol}^{-1}$. Mass spectrometry reveals that the composition consists of approximately $50 \%$ polysorbate monolaurates, with the remainder consisting of myristates, palmitates, and stearates [29]. Older studies measured the CMC of Tween 20 around $0.06 \mathrm{~g} \mathrm{~L}^{-1}$; however, recent studies place the $\mathrm{CMC}$ closer to $0.116 \mathrm{~g} \mathrm{~L}^{-1}$ [30] and $0.135 \mathrm{~g} \mathrm{~L}^{-1}[31,32]$ and the aggregation number between 24 and 29 monomers [33]. The discrepancy is possibly due to changes in the manufacturing of the commercial product or batch-to-batch variation due to the heterogeneous composition [31]. Premicellar diffusion coefficients for Tween 20 measured using the surface pressure technique range from 1.9 to $0.3 \times$ $10^{-10} \mathrm{~m}^{2} \mathrm{~s}^{-1}$ for low concentration to concentrations near the $\mathrm{CMC}$. The decrease in diffusion coefficient with concentration even below the CMC may be due to the increasing presence of dimers in the premicellar solution. The properties of the two surfactants are summarized in Table II.

\section{EXPERIMENTAL OBSERVATIONS}

The experimental study covered a capillary number range of $\mathrm{Ca}=0.003$ to 0.02 with drop formation times from 10 to $200 \mathrm{~ms}$. Within these limits the T-junction generator is expected to operate in the squeezing-to-transition regime [25]. Figure 1 shows the typical drop formation sequence when surfactants are present for both T-junction designs used in experiments. Based on qualitative observations there is no obvious difference in the evolution of the interface as compared to the case when surfactants are absent (refer to [24]).

First, we attempt to quantify the degree to which dynamic interfacial tension influences the droplet formation process. For this purpose, we compare surfactant experimental data with the predictions obtained from the operational model developed in Ref. [24] using the equilibrium interfacial tension in the calculations. Parity plots comparing the experimentally measured dimensionless droplet volume, $V_{d}^{*}=V_{d} / w_{c}^{2} h$, and

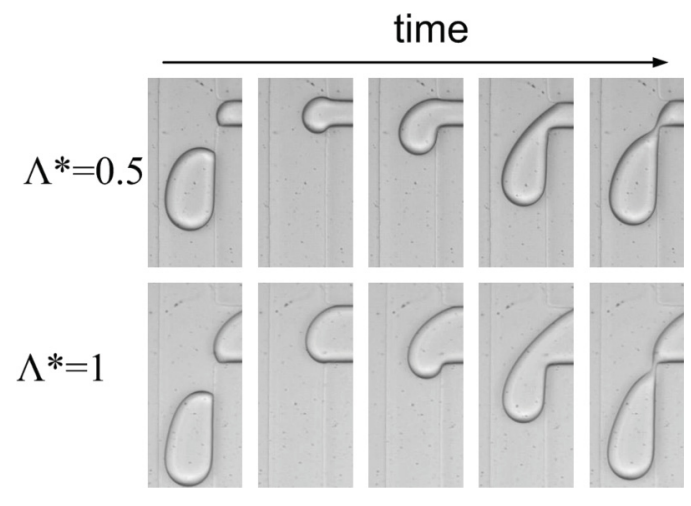

FIG. 1. Series of micrographs showing the formation of droplets in the two T-junction designs $\left(\Lambda^{*}=0.5,1\right)$ with $1.5 \%$ SDS under conditions of $\mathrm{Ca}=0.0115$ and $\varphi=0.373$.

penetration depth in the filling stage, $b_{\text {fill }}^{*}=b_{\text {fill }} / w_{c}$, to the model are presented in Fig. 2. The data show that the model systematically underpredicts the operation of the T-junction generator. Droplets are larger than expected, which suggests that the interfacial tension during formation is greater than the equilibrium value. Also, deviations are more pronounced for solutions of Tween 20 than SDS, as the former has a lower diffusion coefficient than the latter. In addition, for each surfactant, the deviation from the model decreases with increasing surfactant concentration. More importantly, these data show that dynamic interfacial effects must always be considered when modeling microfluidic droplet generators, even for a small, fast adsorbing surfactant such as SDS [20,22].

Droplet formation is associated with a large change in the shape and area of the emerging interface. For an expanding interface the dynamic interfacial tension depends on the dilation rate. In the case of the $\mathrm{T}$-junction generator, the dilation rate is primarily dependent on the flow of the dispersed phase, $Q_{d}$. Figure 3 plots the variation in $b_{\text {fill }}^{*}$ against $Q_{d}$ for a constant surfactant concentration of Tween 20 against nearly constant $\mathrm{Ca}$. The data show that there is a significant correlation between $b_{\text {fill }}^{*}$ and $Q_{d}$ which is not evident when surfactants are absent [24,25].

From Ref. [25], the penetration depth $b_{\text {fill }}^{*}$ can be derived from a force balance on the emerging droplet consisting of a pressure force and an interfacial tension force which resists deformation:

$$
\frac{h^{*}}{\mathrm{Ca}}=\frac{1}{2} \frac{L_{\mathrm{eff}}^{*} g\left(\eta, \lambda_{\mathrm{gap}}\right)}{\left(A_{\mathrm{gap}}^{*} \bar{u}_{\mathrm{gap}}^{*}\right)} \frac{b_{\text {fill }}^{*}}{\left(2-b_{\text {fill }}^{*} / R_{n}^{*}\right)},
$$

where definition of variables are provided in Ref. [25]. This equation is applicable in the squeezing-to-transition regime where droplet formation is dominated by the buildup of pressure behind the interface as it blocks the flow of the continuous phase. It is not applicable in the transition or jetting regimes where the shear force becomes influential.

If $b_{\text {fill }}^{*}$ and the flow conditions are known, then it seems reasonable that Eq. (1) can be rearranged to extract the apparent interfacial tension $\gamma$ at the end of the filling stage from the experimental data:

$$
\frac{\gamma}{\gamma_{\mathrm{eq}}}=\frac{1}{2} \frac{L_{\mathrm{eff}}^{*} g\left(\eta, \lambda_{\mathrm{gap}}\right)}{\left(A_{\mathrm{gap}}^{*} \bar{u}_{\mathrm{gap}}^{*}\right)} \frac{b_{\mathrm{fill}}^{*}}{\left(2-b_{\mathrm{fill}}^{*} / R_{n}^{*}\right)} \frac{\mathrm{Ca}}{h^{*}},
$$




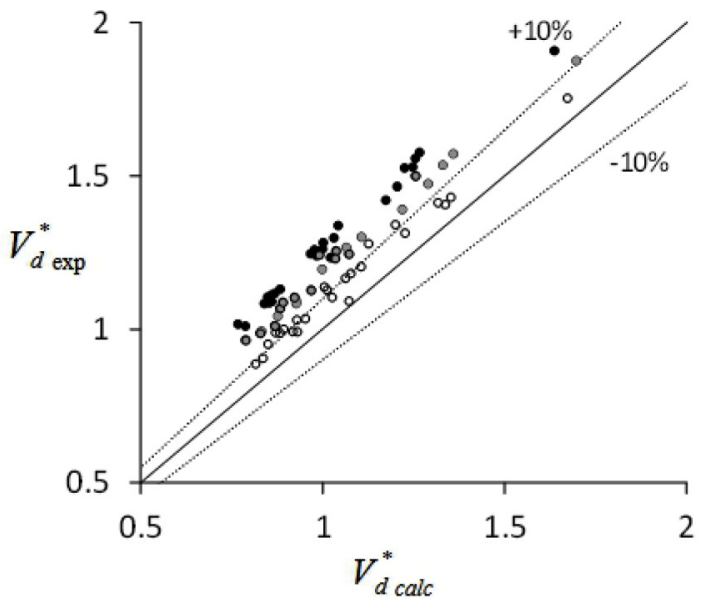

(a)

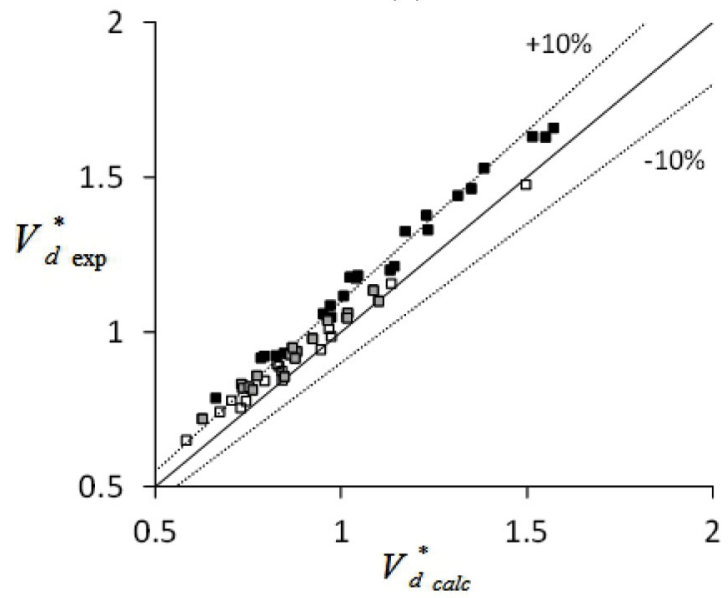

(c)

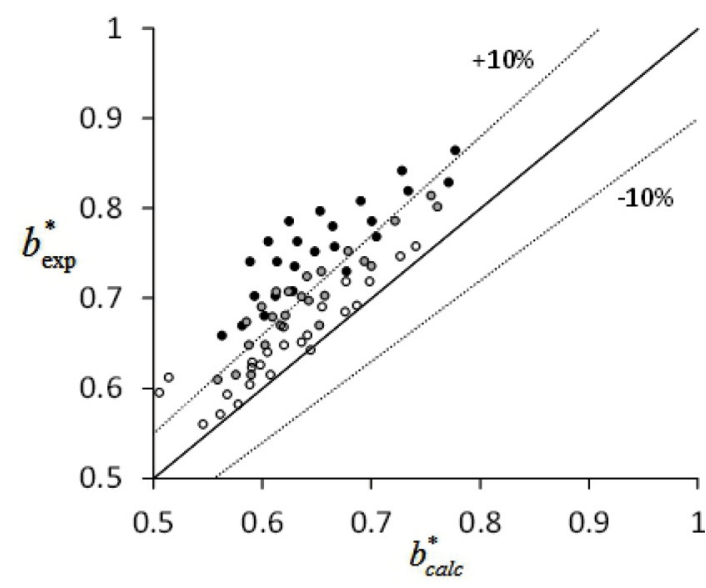

(b)

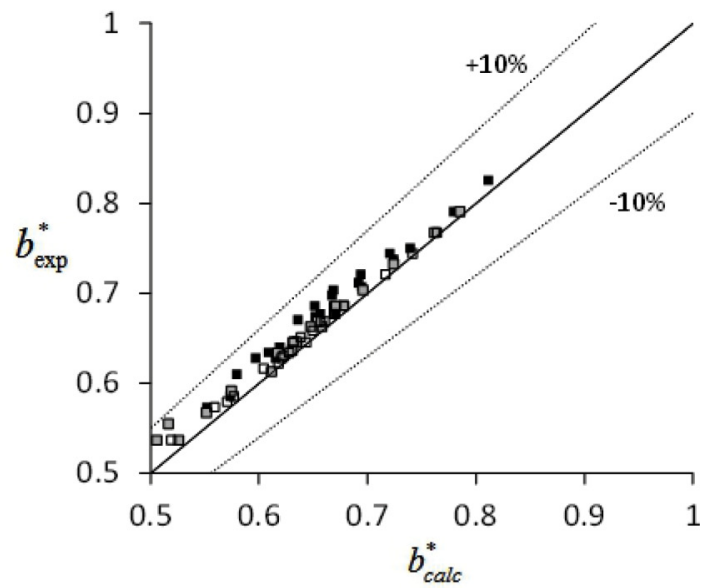

(d)

FIG. 2. Parity plots of the calculated $V_{d}^{*}$ and $b_{\text {fill }}^{*}$ to experimental data using the static interfacial tension. (a) and (b) (o) Tween 20 (black) $0.1 \%$, (gray) $0.5 \%$, and (white) $2 \%$. (c) and (d) ( $\square$ ) SDS (black) $0.5 \%$, (gray) $1.5 \%$, and (white) $3 \%$. Solid black line represents perfect parity and the dotted lines are $\pm 10 \%$.

where $\mathrm{Ca}$ is calculated using the equilibrium interfacial tension.

Before discussing the results of the dynamic interfacial tension measurement we provide a few caveats in the application of Eq. (2). First, Eq. (2) was derived with the condition that the interface acts as a fully mobile interface (slip boundary condition). However, if surfactant coverage is nonuniform, interfacial tension gradients arise which lead to Marangoni stresses that act in opposition to the direction of flow. These gradients may be formed by concentration gradients within the solution or if the dilation rate is not uniform across the interface [3]. Under certain conditions the stresses may cause the interface to act like a solid rather than a liquid (no-slip condition) [1]. If this is the case, then Eq. (1) can be reformulated by developing new expressions for $\bar{u}_{\text {gap }}^{*}$ with the no-slip boundary condition on the interface. However, under high surfactant concentrations and fast adsorption kinetics, gradients are minimal and the interface may remain fully mobile [2,35-38]. Analysis of such effects would require a detailed numerical study that couples surfactant transport with interface deformation and flow of the dispersed and continuous phases $[37,39,40]$. Such an analysis is beyond the scope of this current study; instead, the focus will be on the extent to which the operational model developed previously [25] can be extended to include dynamic interfacial tension effects.

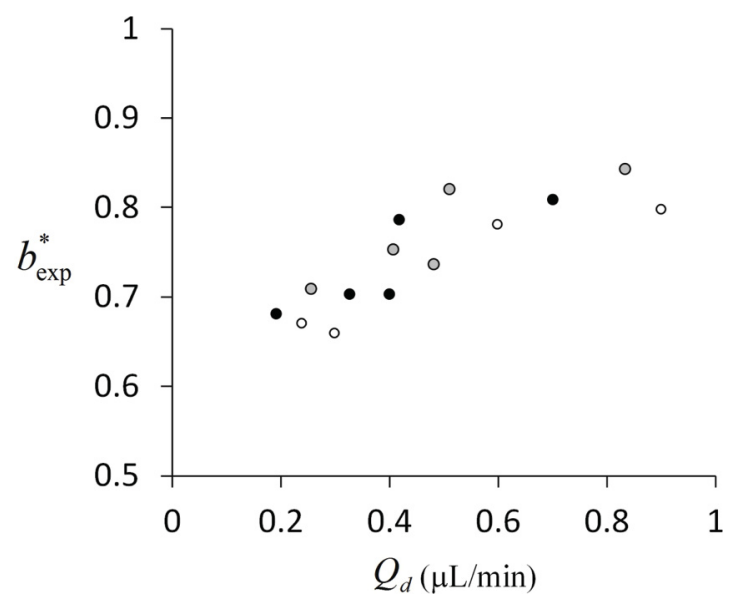

FIG. 3. Variation of $b_{\text {fill }}^{*}$ versus incoming dispersed flow rate $Q_{d}$. Data correspond to T-junction with 1:2 $0.1 \%$ Tween 20 for (black) $Q_{c}=1.5-1.9 \mu \mathrm{L} \mathrm{min}^{-1}$, (gray) $Q_{c}=1.9-2.3 \mu \mathrm{L} \mathrm{min}^{-1}$, and (white) $Q_{c}=2.3-2.8 \mu \mathrm{L} \mathrm{min}^{-1}$. 


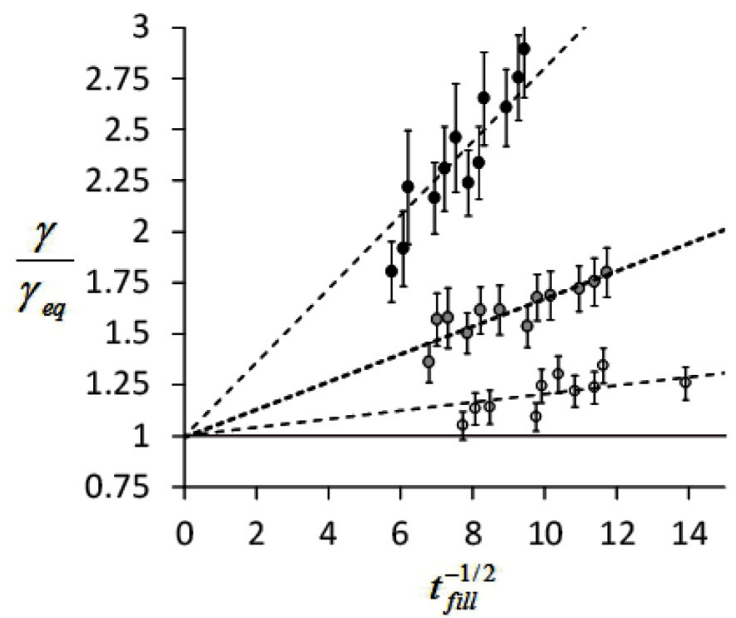

(a)

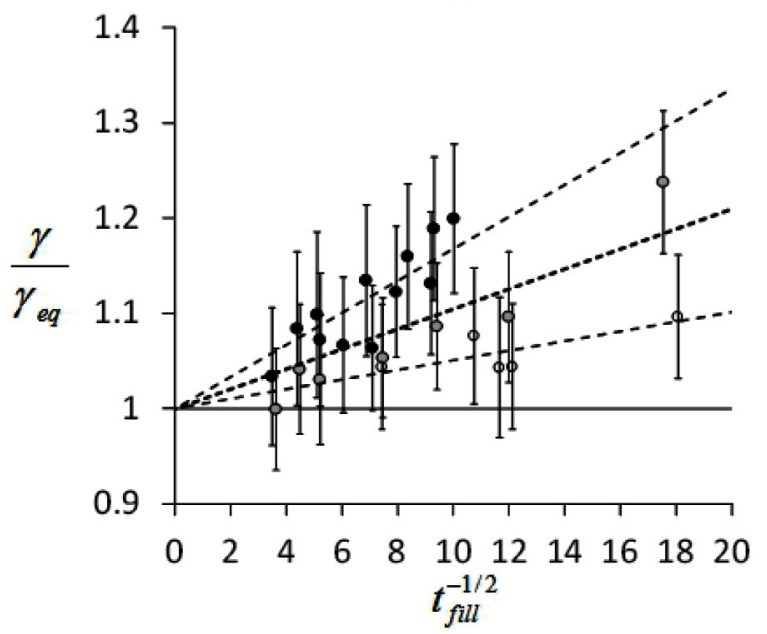

(c)

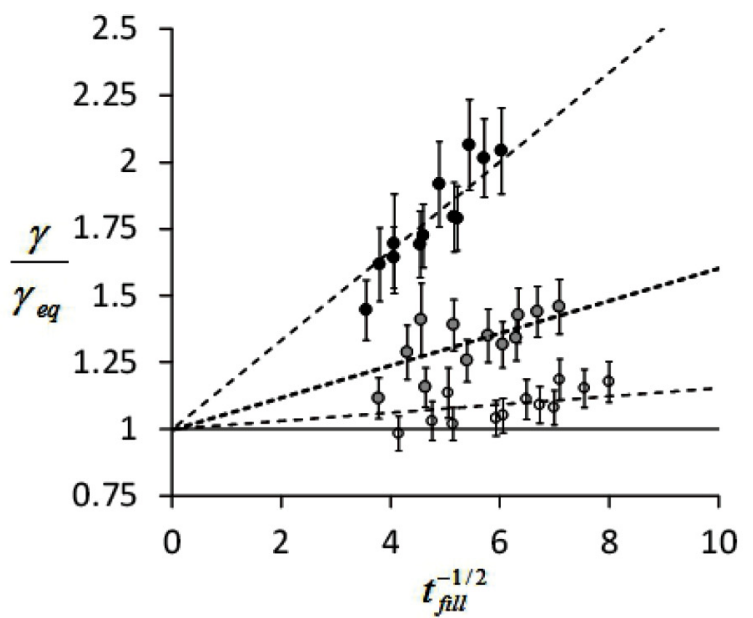

(b)

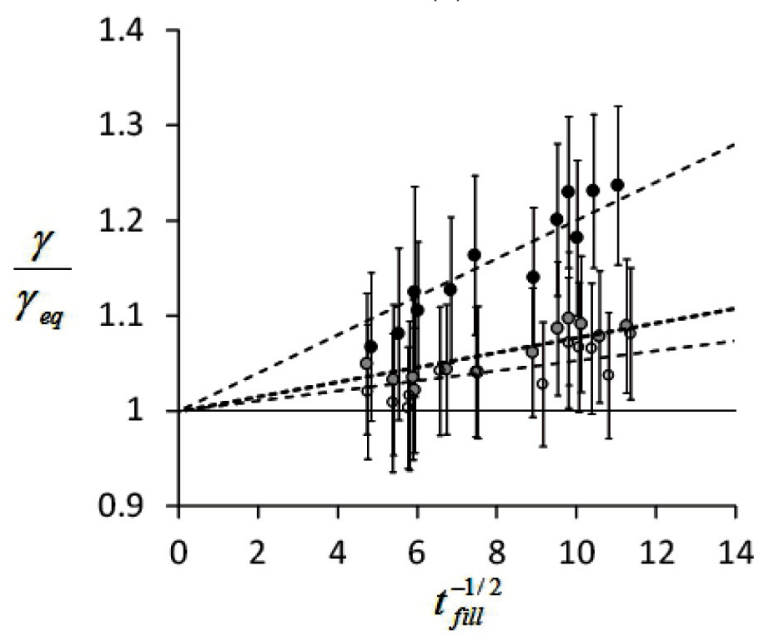

(d)

FIG. 4. Plots of the apparent dynamic interfacial tension extracted from video analysis using Eq. (2). (a) Tween 20 experiments with $\Lambda^{*}=0.5$ T-junction design (black) $0.1 \%$, (gray) $0.5 \%$, and (white) $2 \%$. (b) Tween 20 with $\Lambda^{*}=1$ T-junction design. (c) SDS with $\Lambda^{*}=0.5$ T-junction design (black) $0.5 \%$, (gray) $1.5 \%$, and white (3\%). (d) SDS with the $\Lambda^{*}=1 \mathrm{~T}$-junction design. Error bars are associated with the \pm 1 pixel error for $b_{\text {fill }}^{*}$ measured from the videos.

Figure 4 presents the apparent interfacial tension for Tween 20 and SDS solutions for both T-junction designs as a function of $t_{\text {fill }}^{-1 / 2}$, where $t_{\text {fill }}=V_{\text {fill }} / Q_{d}$. Plotting dynamic interfacial tension data in this manner is common practice since loading of the interface generally scales with $t_{\text {fill }}^{-1 / 2}$ for both diffusive and convective transport of surfactants [2]. It should be stated that the error in the extraction of the interfacial tension is quite significant. Because the relation between $b_{\text {fill }}^{*}$ and $\gamma$ is highly nonlinear $\left(\gamma \propto b_{\text {fill }}^{*^{-3}}\right)$, even small errors in $b_{\text {fill }}^{*}$ propagate into larger errors in $\gamma$. The error in measuring $b_{\text {fill }}$ from videos is estimated to be 1 pixel; the error bars in Fig. 4 represent this estimated error in the calculation of $\gamma$ from Eq. (2).

As expected, the dynamic effects are more pronounced in the Tween 20 solutions as compared to the SDS solutions and increasing the surfactant concentration reduces the dynamic interfacial tension effects. For the lowest concentration of Tween $20(0.1 \%)$, which is still ten times above the CMC, the apparent interfacial tension is somewhere between $150 \%$ and $300 \%$ of the equilibrium value. In fact, the higher end of this range is closer to the bare interface $\sim 38 \mathrm{mN} \mathrm{m}^{-1}$ than to the equilibrium value $\sim 9.3 \mathrm{mN} \mathrm{m}^{-1}$. Even for the lowest concentration of SDS, which is two times the CMC, the apparent interfacial tension is $20 \%$ higher than the equilibrium value. Additionally, for the same surfactant concentration, dynamic interfacial effects are slightly more pronounced in the T-junction design with the smaller inlet $\left(\Lambda^{*}=0.5\right)$ than the large inlet $\left(\Lambda^{*}=1\right)$. This suggests that the design of the T-junction generator plays an important role in the dynamic interfacial tension as well. Based on these observations, a theoretical model was developed to explain the relationship between the various parameters and the dynamic interfacial tension.

\section{MODELING OF SURFACTANT EFFECTS}

The decrease in interfacial tension is directly related to the number of adsorbed surfactant molecules interface as defined by the Gibbs isotherm $[2,41]$ :

$$
\Gamma=-\frac{1}{n R T} \frac{\partial \gamma}{\partial \ln c},
$$


where $\Gamma$ is the excess surface concentration of surfactant (mol $\left.\mathrm{m}^{-2}\right), R$ is the gas constant $\left(8.314 \mathrm{~J} \mathrm{~K}^{-1} \mathrm{~mol}^{-1}\right), T$ the temperature in Kelvin, $\gamma$ the interfacial tension $(\mathrm{N} \mathrm{m})$, and $c$ the concentration of surfactant in the bulk $\left(\mathrm{mol} \mathrm{m}^{-3}\right) ; n=$ 1 for neutral surfactants and $n=2$ for ionic surfactants in the absence of excess electrolytes [41]. This equation can be integrated if the relationship between the surface concentration and the bulk concentration is known for the equilibrium state [41]. Under dynamic conditions the adsorption must be modeled by also including the convective and diffusive transport of surfactant monomers.

Adsorption of surfactants is driven by the concentration gradient created between the subsurface and the bulk as molecules are transferred to the interface [2]. If diffusion is the rate controlling step, then the boundary condition for the conservation of mass at the interface is

$$
\frac{\partial \Gamma}{\partial t}+\Theta \Gamma=D\left(\frac{\partial c}{\partial z}\right)_{o},
$$

where $z$ is the coordinate normal to the surface, $c$ the concentration in the bulk, $D$ the diffusion coefficient, and $\Theta$ the dilation rate. Alternatively, Eq. (4) can be reformulated for cylindrical or spherical coordinate systems [2]; however, the process is usually not necessary as the diffusion penetration depth $l_{D} \sim(\pi D t)^{1 / 2}$ is usually much smaller than the radius of curvature. However, this assumption may fail for droplets of small diameter $(<70 \mu \mathrm{m})$ with low concentrations of surfactant, as the diffusion boundary layer will approach the size of the droplet [42]. Under such conditions, the following derivations need to be modified to account for kinetic controlled adsorption [2,19].

Based on Eq. (4), the difference between the increase in coverage due to surface area changes $\Theta$ and diffusive transport $D(\partial c / \partial z)_{o}$ determines the change in coverage of the interface. For an expanding interface the dilation rate is positive, and thus it represents a depletion of surface coverage in the mass transfer process. When the rate of expansion is high, diffusion is not able to supply the interface with enough surfactant; hence the coverage decreases and the interfacial tension approaches that of the natural oil-water interface. Conversely, at low expansion rates the supply is sufficient and the interfacial tension does not change significantly.

Joos and Vanuffelen demonstrated that Eq. (4) can be integrated using Laplace transformations and arranged into a form similar to the classical Ward and Tordai [43] equation for diffusion $[2,44,45]$ :

$$
\begin{aligned}
\Gamma(\tau) f(t)= & \Gamma_{o}+2\left(\frac{D \tau}{\pi}\right)^{1 / 2} c_{o}-2\left(\frac{D}{\pi}\right)^{1 / 2} \\
& \times \int_{0}^{\sqrt{\tau}} c_{s}(\tau-\lambda) d \sqrt{\lambda}
\end{aligned}
$$

where $c_{o}$ is the bulk concentration and $c_{s}$ the subsurface concentration, $\Gamma_{o}$ the initial surface coverage, $\lambda$ is an integration variable and $f(t)=A(t) / A_{o}$, where $A$ is the interface area and $\tau=\int_{0}^{t} f(t)^{2} d t$. The difficulty associated with solving Eq. (5) analytically is caused by the convolution integral on the right-hand-side which accounts for the back diffusion of surfactants from the interface [46]. For small deviations from equilibrium, Joos and Vanuffelen linearized Eq. (5) under the assumption of a diffusion limited reaction, and found an equation for the jump in interfacial tension based on the "long time" approximation (i.e., close to equilibrium surface coverage) [47]:

$$
\begin{aligned}
\gamma(t)-\gamma_{\mathrm{eq}}= & \Delta \gamma(t)=[f(t)-1]\left[1+\frac{\zeta}{f(t)-1}\right] \\
& \times \frac{n R T \Gamma^{2}}{c} \sqrt{\frac{\pi}{4 \tau D}},
\end{aligned}
$$

where $\zeta=1-\Gamma_{o} / \Gamma_{\mathrm{eq}}$ is the initial surface coverage. If the interface begins at equilibrium then $\Gamma_{o}=\Gamma_{\text {eq }}$, and Eq. (6) reduces to

$$
\Delta \gamma(t)=[f(t)-1] \frac{n R T \Gamma^{2}}{c} \sqrt{\frac{\pi}{4 \tau D}} .
$$

These equations, however, are only valid for submicellar solutions and equations for micellar solutions are needed to compare with experimental conditions in this study. Because of their structure, micelles themselves are not surface active, given that they are unable to straddle the two-phase interface. Only suspended monomers can adsorb to the interface. However, micelles still play an important role in enhancing monomer transport to the interface. At an expanding interface there is a continuous transport of monomers from the bulk to the subsurface as the exposed area increases with time. As a result, there is a local depletion of monomers which breaks the equilibrium between monomers and micelles, causing micelles to break apart, thereby releasing more monomers into the solution [2]. In turn, the disintegration of micelles also creates a local depletion of micelles, and therefore micelles will also diffuse from the bulk into this region as well. Consequently, the diffusion of monomers and micelles is coupled, and the process is described by a chemical reaction that includes the diffusion of each component and the micellization and demicellization kinetics [2].

To account for the contribution of micelles in the surfactant transport, an effective diffusion coefficient that replaces the diffusion coefficient in Eq. (7) is often used based on the assumption that micellization kinetics are fast compared to diffusion [2]:

$$
D_{\text {eff }}=D(1+\beta)\left(1+\beta \sigma^{2}\right),
$$

where $D$ is the diffusion coefficient of the monomers, $\beta=$ $c_{o} / c_{\mathrm{CMC}}-1$ and $\sigma=N_{A}^{-1 / 3}$. The result is that the effective diffusion coefficient is much larger than the component monomers and that the diffusion coefficient increases with increasing concentration of surfactant. Equation (8) is a simplistic representation of the micellization process; more comprehensive models account for the polydispersity of the micelles and the many different pathways of the micellization reaction $[7,48,49]$. Still, it serves as an adequate depiction for a first approximation.

Modifications to the interfacial tension jump to include micelles result in

$$
\Delta \gamma(t)=[f(t)-1] \frac{R T \Gamma_{\mathrm{CMC}}^{2}}{c_{\mathrm{CMC}}} \sqrt{\frac{\pi}{4 \tau D_{\mathrm{eff}}}},
$$

where $c_{\mathrm{CMC}}$ is the concentration and $\Gamma_{\mathrm{CMC}}$ the surface coverage at the CMC. For micellar solutions these parameters 


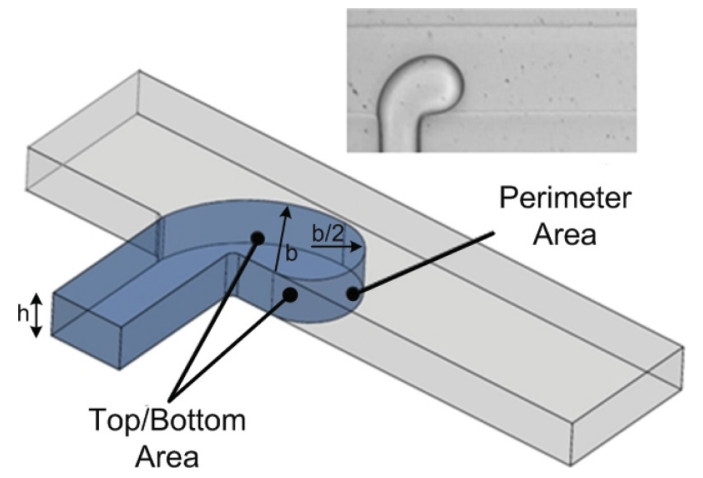

FIG. 5. (Color online) Diagram of the approximated area of the droplet from the projection of the two-dimensional image.

are constant above the CMC and the only parameter that changes with added concentration of surfactant is the effective diffusion coefficient. In order to solve Eq. (9) for the T-junction generator, an expression for the temporal evolution of the interfacial area $A(t)$ is still needed. Such an expression for the T-junction generator is derived next.

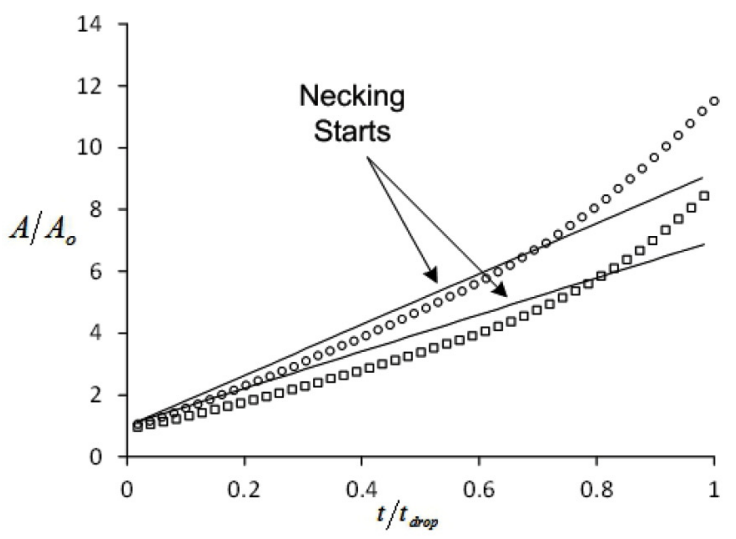

(a)

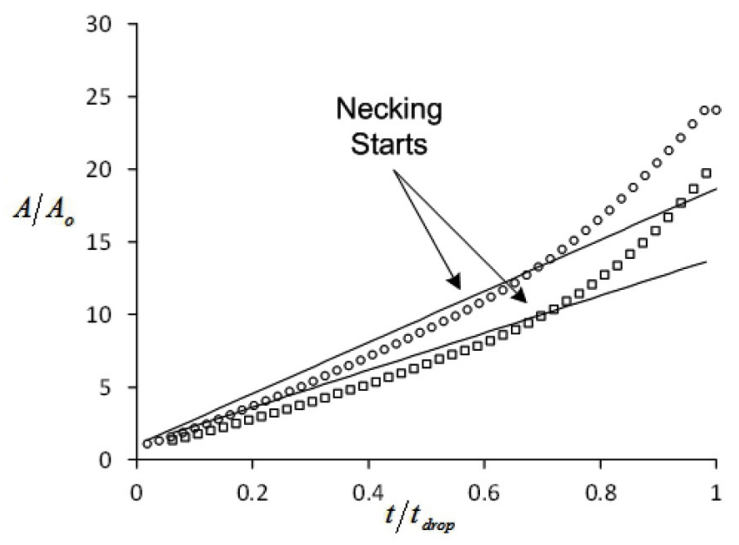

(c)

\section{A. Approximation for $A(t)$ for the $T$ junction}

The area of the emerging interface during the filling stage is approximated using the definitions provided in Fig. 5. The shape of the interface is described as two parts with the back half defined by a quarter circle of radius $b$, and the front half by a half circle of radius $b / 2$. In the following analysis, out of plane curvature effects are neglected for the purpose of simplifying the problem.

Assuming that the flow rate remains constant throughout the formation process, the volume of the emerging droplet is given as

$$
V(t)=\frac{3 \pi}{8} b(t)^{2} h=Q_{d} t .
$$

In the representation given in Fig. 5, the surface area of the drop consists of the top and bottom surfaces and the area defined by the perimeter:

$$
A(t)=2 \frac{3 \pi}{8} b(t)^{2}+\pi h b(t)+A_{o},
$$

where $A_{o}$ is the initial area of the interface before the onset of expansion which, for T-junction designs, may be approximated as the cross-sectional area of the dispersed channel $A_{o}=$ $w_{d} h$. In a previous derivation by van der Graaf et al., the

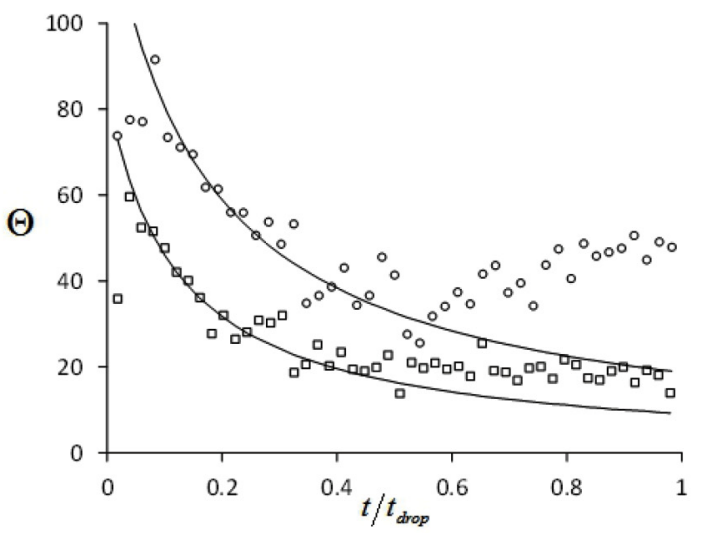

(b)

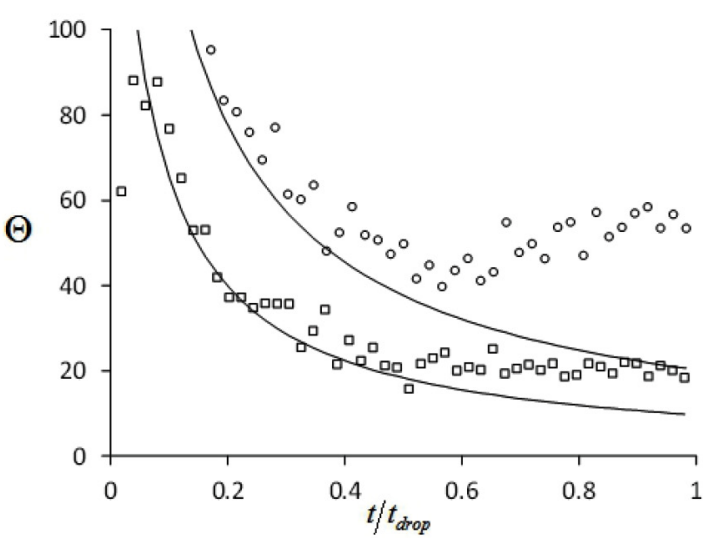

(d)

FIG. 6. Area change and dilation rate extracted from video analysis of droplet formation. (a) and (b) T-junction $\Lambda^{*}=0.5, h^{*}=0.5$ with silicone oil and $1.5 \%$ SDS water with conditions of $(\square) \mathrm{Ca}=0.049, b_{\text {fill }}^{*}=0.79, f=17.8 \mathrm{~Hz}$. (०) $\mathrm{Ca}=0.015, b_{\text {fill }}^{*}=0.645, f=42.8 \mathrm{~Hz}$. (c) and (d) T-junction $\Lambda^{*}=1, h^{*}=0.5$ with silicone oil and $0.5 \%$ SDS water with conditions of ( $\square$ ) $\mathrm{Ca}=0.0048, b_{\text {fill }}^{*}=0.741, f=9.66 \mathrm{~Hz}$. (०) $\mathrm{Ca}=0.0149, b_{\text {fill }}^{*}=0.598, f=20.9 \mathrm{~Hz}$. Solid black lines are calculations from Eqs. (15) and (16). 
top and bottom areas were neglected under the assumption that exposure to the surfactant occurs primarily along the perimeter [21]. Here the top and bottom surfaces are included because surfactant was added to the dispersed phase instead of the continuous phase where absorption is expected to occur at all exposed surfaces.

Substituting Eq. (10) into Eq. (11) the change in area as a function of time is

$$
A(t)=A_{o}\left[1+f_{1} t+f_{2} t^{1 / 2}\right] .
$$

The dilation rate is given as $\Theta=[1 / A(t)](\partial A / \partial t)$, where upon substitution of the previous equations,

$$
\Theta=\frac{\left(f_{1}+\frac{f_{2}}{2} t^{-1 / 2}\right)}{1+f_{1} t+f_{2} t^{1 / 2}},
$$

with $f_{1}=2 \frac{Q_{d}}{A_{o} h}$ and $f_{2}=\frac{1}{A_{o}}\left(\frac{8 \pi}{3} Q_{d} h\right)^{1 / 2}$.

To help simplify the problem, the term $f_{2} t^{1 / 2}$ can be linearized using a first order Taylor expansion around time $t_{o}$ :

$$
f_{2} t^{1 / 2} \approx \frac{f_{2}}{2 t_{o}^{1 / 2}} t=\left(\frac{8 \pi}{3} Q_{d} h\right)^{1 / 2} \frac{t}{2 A_{o} t_{o}^{1 / 2}}
$$

For convenience, $t_{o}=1 / f_{1}$ is chosen as the characteristic time, and upon substitution the area change and dilation rate becomes

$$
\begin{aligned}
A(t) & =A_{o}\left[1+f_{3} t\right], \\
\Theta & =\frac{f_{3}}{1+f_{3} t},
\end{aligned}
$$

where $f_{3}=f_{1}\left[1+\left(h^{2} / A_{o}\right)^{1 / 2}\right]$.

To verify these approximations, Eqs. (15) and (16) are plotted against experimental data as shown in Fig. 6. Data are plotted for high and low capillary number conditions for both T-junction designs. One can see that the surface area of the droplet increases at a linear rate in the filling stage and then accelerates during the necking stage. Furthermore, the dilation rate decreases quickly during the filling stage decaying to a constant value in the necking stage. The fits provided by Eqs. (15) and (16) agree well with the experimental data, especially in the filling stage for the dilation rate where we are most interested in capturing the interface expansion effects.

The interfacial tension jump can then be calculated for a T-junction generator:

$$
\Delta \gamma(t)=\frac{n R T \Gamma_{\mathrm{CMC}}^{2}}{c_{\mathrm{CMC}}} \sqrt{\frac{3 \pi}{4 D_{\mathrm{eff}}}} \sqrt{\frac{f_{3}^{2} t}{\left(f_{3} t\right)^{2}+3 f_{3} t+3}} .
$$

Equation (17) demonstrates that for a given surfactant $\left(c_{\mathrm{CMC}}\right.$, $\left.D_{\text {eff }}, \Gamma_{\mathrm{CMC}}\right)$, the dynamic interfacial tension depends on the shape of the T-junction generator $\left(A_{o}, h\right)$ and the flow rate of the dispersed phase $\left(Q_{d}\right)$ in agreement with experimental observations described previously. One should note that in applying Eq. (17) it is assumed that the interface is at equilibrium before expansion starts. In reality, this condition may not be met in a droplet generator when droplets are formed at high rates and the interface does not have time to rest. It should also be stated that the analysis has not included additional transport effects that might enhance adsorption such as internal circulation within the droplet. These two effects are difficult to quantify within an analytical model, but should be recognized as having a potential influence on the dynamic interfacial tension.

\section{B. Model validation with experimental data}

The data from both T-junction generators should collapse onto the same curve when plotted against the temporal term in Eq. (17) as the slope is dependent only on the properties of the surfactant. Figure 7 confirms this hypothesis, indicating that the equation developed for $A(t)$ is correct.

Next, the slopes from these curves can be used to compare with the estimated slopes given by the surfactant properties

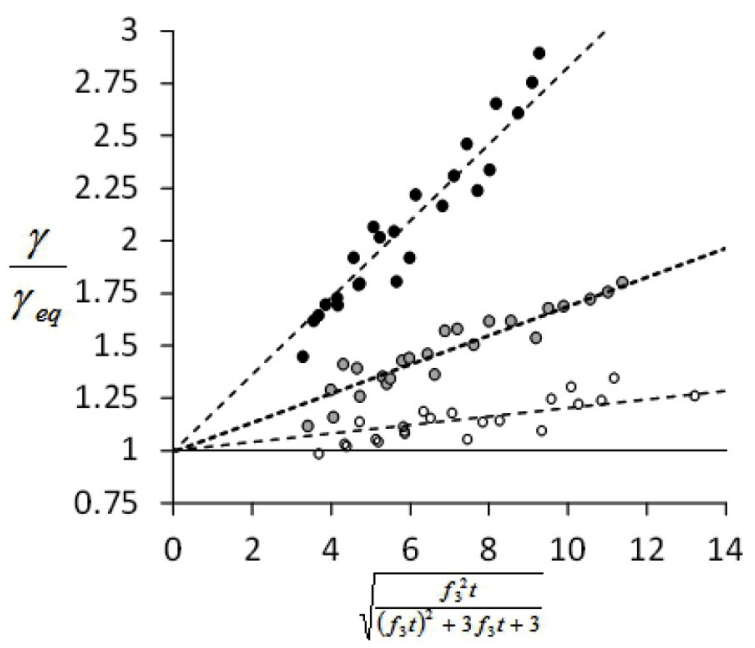

(a)

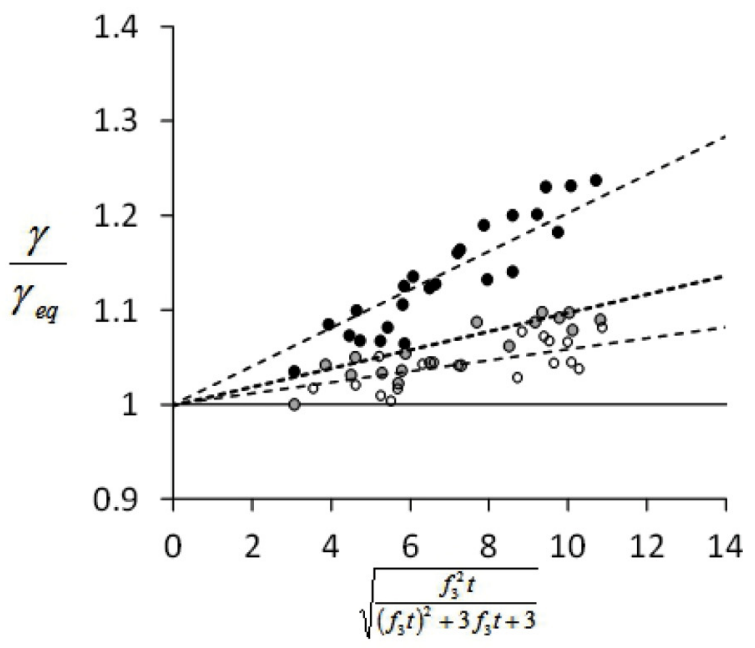

(b)

FIG. 7. Plot of data from both 1:2 and 1:1 T-junction designs for the dynamic interfacial tension versus the temporal term in Eq. (17). (a) Tween 20 with (black) $0.1 \%$, (gray) $0.5 \%$, and (white) $2 \%$. (b) SDS (black) $0.5 \%$, (gray) $1.5 \%$, and (white) $3 \%$. Dashed lines are linear fits to the data used to extract the slope. Solid line represents an interfacial tension equal to the static interfacial tension. Error bars are omitted for clarity but are the same scale as those in Fig. 4. 
TABLE III. Comparison of slopes extracted from Fig. 5.10 and the range calculated from Eq. (5.19) using surfactant properties found in the literature.

\begin{tabular}{lll}
\hline \hline Surfactant & Slope & $\frac{n R T \Gamma_{\mathrm{CMC}}^{2}}{{ }^{{ }_{\mathrm{CMC}}} \sqrt{\frac{3 \pi}{4 D_{\mathrm{eff}}}}}$ \\
\hline Tween 20, 0.1\% & 0.202 & $0.1800-0.5000$ \\
Tween 20, 0.5\% & 0.0731 & $0.0379-0.1054$ \\
Tween 20, 2\% & 0.0204 & $0.00958-0.0266$ \\
SDS, 0.5\% & 0.0203 & $0.0158-0.0282$ \\
SDS, 1.5\% & 0.0098 & $0.0078-0.0154$ \\
SDS, 3\% & 0.0059 & $0.00683-0.0121$ \\
\hline \hline
\end{tabular}

listed previously in Table II. Table III provides the comparison between the measured and calculated slopes. As part of these calculations a typical surface excess coverage of $\Gamma_{e}=(3-4) \times$ $10^{-6} \mathrm{~mol} \mathrm{~m}^{-2}$ was used for SDS and (1.5-2.5) $\times 10^{-6} \mathrm{~mol}$ $\mathrm{m}^{-2}$ for Tween 20 [50]. The measured slopes fall within the anticipated range calculated from the surfactant properties, though the calculated range is rather large because of the ambiguity surrounding many of the surfactant properties.

The analysis has shown that the theory developed herein can be used to predict the influence of dynamic interfacial tension on the droplet formation process. One can understand the influence of surfactant properties, T-junction design, and operating conditions on the dynamic interfacial tension.

\section{Modification to the droplet formation model}

In this section, this new model for the dynamic interfacial tension is adapted to the model for droplet generation in Paper II; please refer to [25] for details on the original model. For the calculation of $b_{\text {fill }}^{*}$, the dynamic interfacial tension is updated at each iteration step until convergence occurs for the force balance. Next, the formation time is calculated as $t=V_{d} / Q_{d}$, where $Q_{d}$ is known, and the dynamic interfacial tension is calculated from Eq. (17). This value is then substituted into the force balance to check if convergence is met. If not, then $b_{\text {fill }}^{*}$ is increased by a small amount and the process repeats.

Calculation of the pinch-off point also includes the interfacial tension as a parameter; however, due to extensive coupling between the final moment of pinch-off, and the ratio of the two flow rates that determine the necking period it is difficult to predict the dynamic interfacial tension at pinch-off. For simplicity, the dynamic interfacial tension is calculated from Eq. (17) with $t_{\text {neck }}=2 t_{\text {fill }}$ and then this value is substituted into the calculation for the critical pinch-off point. Without further modifications the amalgamated model was used to predict the operation of the T-junction generator using the slopes obtained in Table III.

Figure 8 shows the parity plot for droplet volume predicted using the modified model. Even with the addition of the dynamic interfacial tension the model still underpredicts the droplet volume with a large degree of error $(\sim 10-20 \%)$. A detailed investigation revealed that the filling stage was indeed accurately predicted by the model, and that the error was

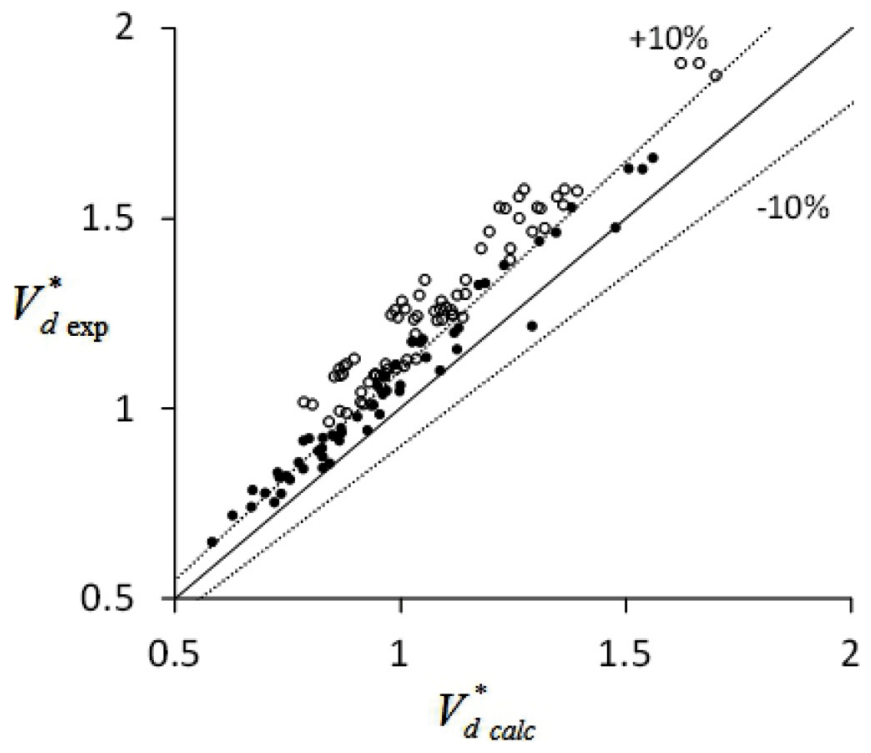

FIG. 8. Parity plot of the droplet volume calculated using the dynamic interfacial tension in the amalgamated model. (o) Tween 20 experimental data; $(\bullet)$ SDS experimental data.

caused by an underprediction of the necking stage due to two reasons.

First, the critical neck thickness for pinch-off, $2 r_{\text {pinch }}$, was smaller than predicted in Ref. [25]. A smaller neck thickness at pinch-off corresponds to longer overall necking periods. It is believed that the longer necking time is caused by the presence of Marangoni stresses due to the depletion of surfactants near the neck during the final moments of collapse where area changes are severe. These stresses retard the drainage of the inner fluid from the thread, thus prolonging the necking stage in comparison to when surfactants are absent. Similar observations have been reported in microfluidic flow focusing devices during necking in the presence of surfactants [51-53].

Second, the back half of the droplet experiences a greater degree of deformation when surfactants are present. Figure 9 presents an example of the disagreement between the previous geometric descriptions of the droplet compared to the experimental observations. When surfactants are absent the back half of the droplet follows the circular segment approximation very well (refer to top of Fig. 9). However, when surfactants are present the neck becomes more elongated and "flattened" as it approaches the inner corner of the T junction. Similar observations of neck "stretching" have been noted by other researchers when the interfacial tension is very low in T-junction generators [20,21]. The fatter neck may be caused by interfacial tension gradients on the back half of the droplet because of dilation differences across the interface as the neck collapses.

As a result, the geometric description that was used in the previous model [25] does not accurately describe the shape of the neck during collapse. This error may be corrected by including the missing area in the calculation of the neck shape. Figure 9 presents the diagram with the geometric parameters that can be used to calculate the missing area. The area is calculated from the difference between the circular segment 

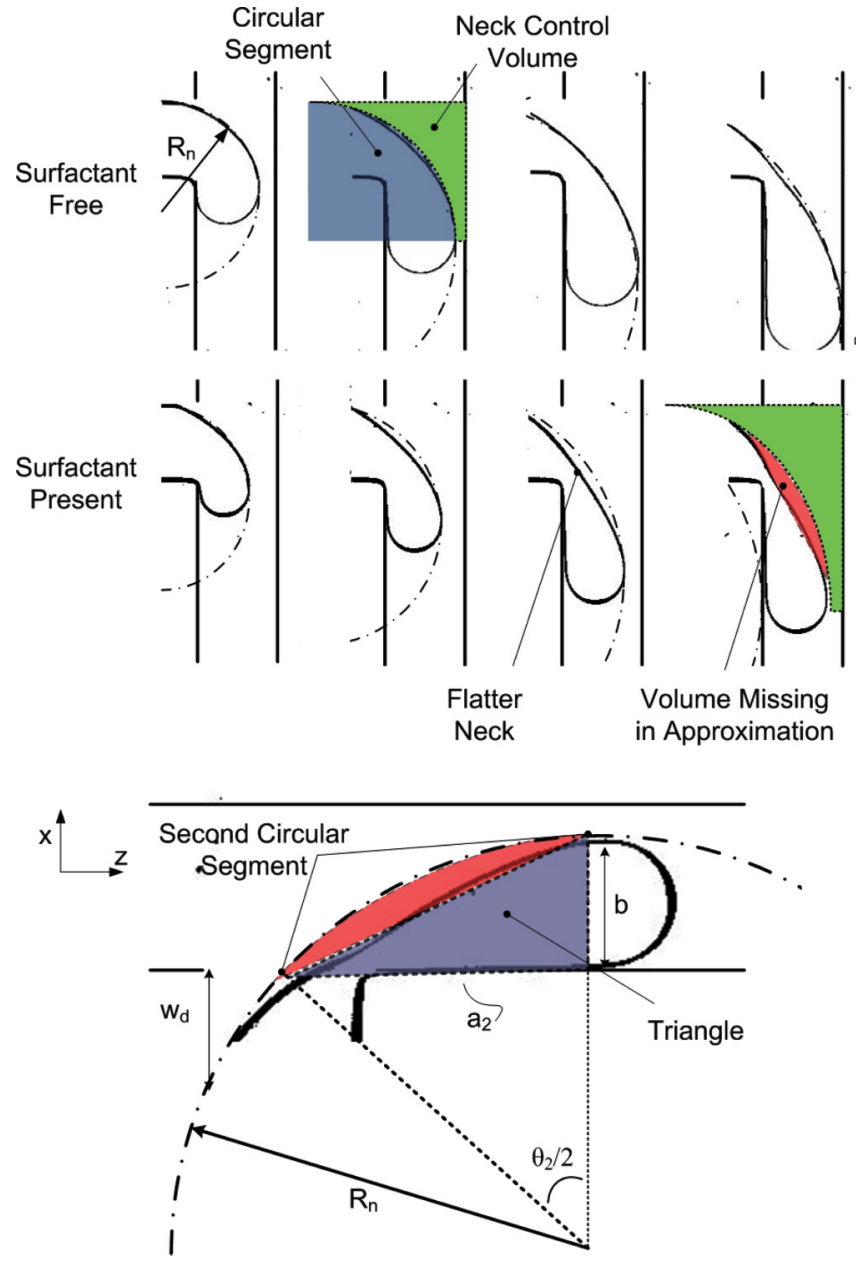

FIG. 9. (Color online) (Top) A sequence of traces showing the boundary of the droplet as it evolves during the necking stage with and without surfactants. For the surfactant free case the back half of the droplet follows the circular segment approximation (highlighted in blue). With surfactants the neck shape diverges from a circle and appears flatter. The volume missing from the approximation is highlighted in red. (Bottom) A diagram showing the geometric parameters that can be used to calculate the missing volume.

and the triangle in Fig. 9:

$$
\begin{aligned}
d_{2}^{*} & =b_{2}^{*}, \quad a_{2}^{*}=\sqrt{d^{*}\left(2 R_{\text {pinch }}^{*}-d^{*}\right)}, \\
\theta_{2} & =2 \arccos \left(1-\frac{b_{2}^{*}}{R_{\text {pinch }}^{*}}\right), \\
A_{\text {mod }}^{*} & =\frac{R_{\text {pinch }}^{* 2}}{4}\left(\theta_{2}-\sin \theta_{2}\right)-\frac{1}{2} a_{2}^{*} b_{2}^{*},
\end{aligned}
$$

where all variables are nondimensionalized by the channel width $w_{c}$. The new neck pinch-off volume may be calculated as

$$
\begin{aligned}
V_{c_{\text {pinch }}}^{*}= & \left(1-b_{2}^{*}\right) a^{*}+\left(1+\Lambda^{*}\right) a^{*}-\frac{R_{\mathrm{pinch}}^{*^{2}}}{4}(\theta-\sin \theta) \\
& +A_{\text {mod }}^{*}+\frac{h^{*}}{2}\left(1-\frac{\pi}{4}\right) R_{\text {pinch }}^{*} \frac{\theta}{2},
\end{aligned}
$$

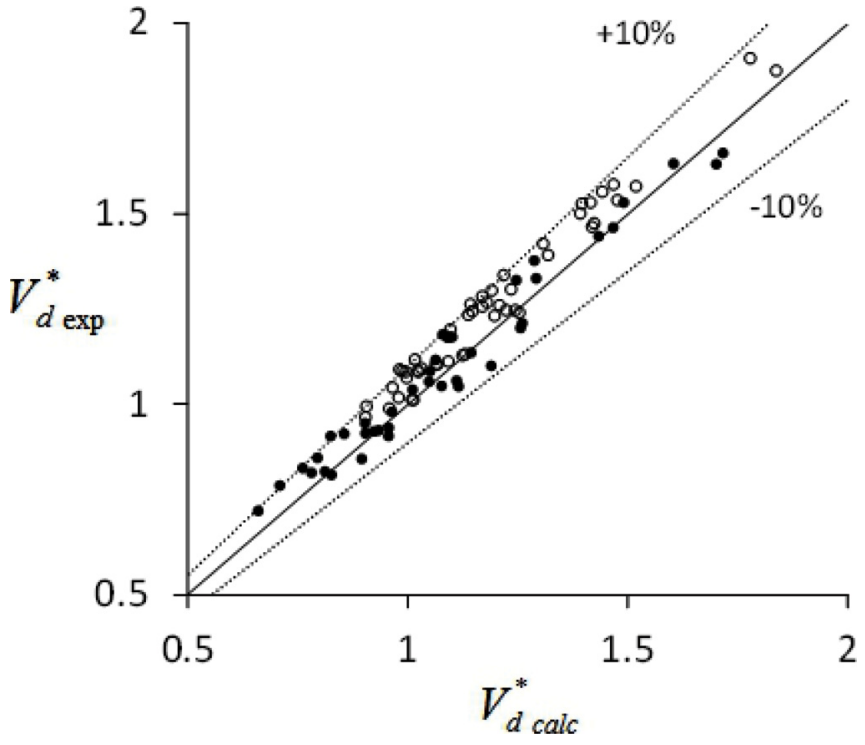

FIG. 10. Parity plot of the droplet volume calculated using the modifications to the neck volume calculation. (o) Tween 20 experimental data; $(\bullet)$ SDS experimental data.

where $V_{c_{\text {pinch }}}^{*}$ is used to calculate the dimensionless necking parameter:

$$
\beta=\frac{1}{\left(1-\frac{A_{\text {gap }}^{*}}{h^{*}}\right)}\left(V_{c_{\text {pinch }}}^{*}-V_{c_{\text {fill }}}^{*}\right) .
$$

Figure 10 presents the comparison of experimental data to the new model that includes the dynamic interfacial tension, critical neck thickness for pinch-off, and neck volume at pinch-off modifications. The data now fall within $\pm 10 \%$ of the predicted values, which is deemed to be acceptable considering the estimated uncertainty of the various parameters. Therefore, the original model for the T-junction generator has been successfully extended to include dynamic interfacial tension effects.

Revisiting the original comparison between the operational model and the experimental data (Fig. 2), we saw that the largest error in predicting the droplet size was only $20 \%$. Considering that this data corresponds to $0.1 \%$ Tween 20 , where the dynamic interfacial tensions increased by 50 $300 \%$ above equilibrium, it seems reasonable that to a first approximation the equilibrium interfacial tension can simply be used. The reason for the weak dependence of $V_{d}$ with $\gamma$ is that effects are dampened because $V_{d}$ scales approximately as $\gamma^{1 / 3}$. If a more accurate calculation is required then the dynamic interfacial tension effects can be included through the interfacial tension jump approximation.

\section{CONCLUSION}

This study examined the influence of surfactants on droplet formation in microfluidic T-junction generators. With microfluidic droplet generators formation times and surfactant adsorption occur at the same time scale resulting in a dynamic system where the droplet formation process is coupled with surfactant transport through the temporally varying interfacial tension. The importance of these effects was 
clearly demonstrated in this study. A model was developed to estimate the jump in interfacial tension during the formation process for surfactant solutions above and below the CMC. As part of this model, a new derivation for the relative interface expansion rate in microfluidic $\mathrm{T}$ junctions was also developed. Based on these equations one can understand the relationship between surfactant properties, T-junction design, and operating conditions on the dynamic interfacial tension.
The adsorption kinetics model was then incorporated into the previous model that describes overall generator performance. Small modifications were needed to the performance model due to deviations in the limit of pinch-off and increased deformation of the droplet neck. Overall, the new model can effectively predict generator performance when surfactants are present and can be used to design and operate T-junction generators.
[1] J. C. Baret, Lab. Chip 12, 422 (2011).

[2] P. Joos, Dynamic Surface Phenomena (VSP BV, Zeist, Netherlands, 1999).

[3] P. Erni, Soft Matter 7, 7586 (2011).

[4] J. C. Earnshaw, E. G. Johnson, B. J. Carroll, and P. J. Doyle, J. Colloid Interface Sci. 177, 150 (1996).

[5] R. Miller, M. Bree, and V. B. Fainerman, Colloid Surf., A 142 237 (1998).

[6] R. Miller, S. A. Zholob, A. V. Makievski, P. Joos, and V. B. Fainerman, Langmuir 13, 5663 (1997).

[7] N. C. Christov, K. D. Danov, P. A. Kralchevsky, K. P. Ananthapadmanabhan, and A. Lips, Langmuir 22, 7528 (2006).

[8] R. L. Bendure, J. Colloid Interface Sci. 35, 238 (1971).

[9] J. Kloubek, J. Colloid Interface Sci. 41, 1 (1972).

[10] C. A. Macleod and C. J. Radke, J. Colloid Interface Sci. 160, 435 (1993).

[11] X. G. Zhang, M. T. Harris, and O. A. Basaran, J. Colloid Interface Sci. 168, 47 (1994).

[12] S. M. Joscelyne and G. Tragardh, J. Membr. Sci. 169, 107 (2000).

[13] C. Charcosset, I. Limayem, and H. Fessi, J. Chem. Tech. Biotech. 79, 209 (2004).

[14] S. van der Graaf, C. Schroen, R. G. M. van der Sman, and R. M. Boom, J. Colloid Interface Sci. 277, 456 (2004).

[15] V. Schroder, O. Behrend, and H. Schubert, J. Colloid Interface Sci. 202, 334 (1998).

[16] S. Sugiura, M. Nakajima, T. Oda, M. Satake, and M. Seki, J. Colloid Interface Sci. 269, 178 (2004).

[17] M. Rayner, G. Tragardh, and C. Tragardh, Colloid Surf., A 266, 1 (2005).

[18] M. J. Geerken, R. G. H. Lammertink, and M. Wessling, J. Colloid Interface Sci. 312, 460 (2007).

[19] J. C. Baret, F. Kleinschmidt, A. El Harrak, and A. D. Griffiths, Langmuir 25, 6088 (2009).

[20] M. L. J. Steegmans, A. Warmerdam, K. Schroen, and R. M. Boom, Langmuir 25, 9751 (2009).

[21] S. van der Graaf, M. L. J. Steegmans, R. G. M. van der Sman, C. Schroen, and R. M. Boom, Colloid Surf., A 266, 106 (2005).

[22] K. Wang, Y. C. Lu, J. H. Xu, and G. S. Luo, Langmuir 25, 2153 (2009).

[23] N.-T. Nguyen, S. Lassemono, F. A. Chollet, and C. Yang, IEEE Sensors 7, 692 (2007).

[24] T. Glawdel, C. Elbuken, and C. L. Ren, Phys. Rev. E 85, 016323 (2012).
[25] T. Glawdel, C. Elbuken, and C. L. Ren, Phys. Rev. E 85, 016322 (2012).

[26] N. Mikati and S. Wall, Langmuir 9, 113 (1993).

[27] Y. F. Zuev, R. K. Kurbanov, B. Z. Idiyatullin, and O. G. Us'yarov, Colloid J. 69, 444 (2007).

[28] B. L. Bales, L. Messina, A. Vidal, M. Peric, and O. R. Nascimento, J. Phys. Chem. B 102, 10347 (1998).

[29] F. O. Ayorinde, S. V. Gelain, J. H. Johnson, and L. W. Wan, Rapid Commun. Mass Spectrom. 14, 2116 (2000).

[30] H.-C. Mahler, M. Printz, R. Kopf, R. Schuller, and R. Mueller, J. Pharm. Sci. 97, 764 (2008).

[31] R. R. Nino and J. M. R. Patino, J. Am. Oil Chem. Soc. 75, 1241 (1998).

[32] D. Wu, G. Y. Xu, J. Liu, and Y. M. Li, J. Dispersion Sci. Technol. 27, 523 (2006).

[33] K. J. Tiefenbach, H. Durchschlag, and R. Jaenicke, in Analytical Ultracentrifugation $V$, edited by H. Cölfen (Springer, Berlin, 1999), Vol. 113, pp. 135-141.

[34] L. S. C. Wan and P. F. S. Lee, J. Pharm. Sci. 63, 136 (1974).

[35] K. J. Stebe, S. Y. Lin, and C. Maldarelli, Phys. Fluids A 3, 3 (1991).

[36] K. J. Stebe and C. Maldarelli, J. Colloid Interface Sci. 163, 177 (1994).

[37] C. D. Eggleton and K. J. Stebe, J. Colloid Interface Sci. 208, 68 (1998).

[38] J. D. Martin and S. D. Hudson, New J. Phys. 11, 115005 (2009).

[39] S. van der Graaf, T. Nisisako, C. Schroen, R. G. M. van der Sman, and R. M. Boom, Langmuir 22, 4144 (2006).

[40] R. G. M. van der Sman and S. van der Graaf, Rheol. Acta 46, 3 (2006).

[41] J. Eastoe and J. S. Dalton, Adv. Colloid Interface Sci. 85, 103 (2000).

[42] F. Jin, R. Balasubramaniam, and K. J. Stebe, J. Adhes. 80, 773 (2004).

[43] A. F. H. Ward and L. Tordai, J. Chem. Phys. 14, 453 (1946).

[44] P. Joos and M. Vanuffelen, J. Colloid Interface Sci. 155, 271 (1993).

[45] M. Vanuffelen and P. Joos, J. Colloid Interface Sci. 158, 452 (1993).

[46] J. Eastoe, J. S. Dalton, P. G. A. Rogueda, E. R. Crooks, A. R. Pitt, and E. A. Simister, J. Colloid Interface Sci. 188, 423 (1997).

[47] P. Joos and M. Vanuffelen, J. Colloid Interface Sci. 171, 297 (1995). 
[48] V. B. Fainerman, V. D. Mys, A. V. Makievski, J. T. Petkov, and R. Miller, J. Colloid Interface Sci. 302, 40 (2006).

[49] B. A. Noskov, Adv. Colloid Interface Sci. 95, 237 (2002).

[50] V. B. Fainerman, D. Mobius, and R. Miller, Surfactants: Chemistry, Interfacial Properties and Applications (Elsevier Science BV, Amsterdam, 2001).
[51] W. Lee, L. M. Walker, and S. L. Anna, Macromol. Mater. Eng. 296, 203 (2011).

[52] F. Jin, N. R. Gupta, and K. J. Stebe, Phys. Fluids 18, 022103 (2006).

[53] M. Roche, M. Aytouna, D. Bonn, and H. Kellay, Phys. Rev. Lett. 103, 264501 (2009). 